\title{
Finite Element Modeling and Static Strength Analysis on Structure Strength of the High-Speed Maglev Bogie
}

\author{
Xu Shimeng ${ }^{1}$, Li Cen ${ }^{1}$, Yao Yijing ${ }^{1}$, Yuan Yuqing ${ }^{2}$, Li Weiya ${ }^{2}$, Li Qiang ${ }^{1}$ \\ ${ }^{1}$ School of Mechanical, Electronic and Control Engineering, Beijing Jiaotong University, Beijing, China \\ ${ }^{2}$ CRRC Qingdao Sifang Co. LTD, Qingdao, China
}

Email address:

18140625@bjtu.edu.cn (Xu Shimeng),13522998763@139.com (Li Cen)

\section{To cite this article:}

Xu Shimeng, Li Cen, Yao Yijing, Yuan Yuqing, Li Weiya, Li Qiang. Finite Element Modeling and Static Strength Analysis on Structure Strength of the High-Speed Maglev Bogie. International Journal of Transportation Engineering and Technology.

Vol. 5, No. 4, 2019, pp. 92-96. doi: 10.11648/j.ijtet.20190504.15

Received: November 3, 2019; Accepted: November 29, 2019; Published: December 9, 2019

\begin{abstract}
Maglev train with no mechanical contact of the innovative technology, as well as a series of excellent economic and environmental advantages came into being. More and more people pay attention to it, and it has become one of the most promising transportation means in the new century. According to the actual operation of maglev train, a boundary constraint method is proposed in this paper. On the basis of the actual operation of maglev train, a method of boundary constraint is proposed, and the finite element model is established by using HyperMesh software. Afterwards using ANSYS analysis software to analyzed the statics performance of levitation chassis under the four working conditions of maglev vehicle, and static strength of the levitation chassis based on von Mises stress was assessed. In accordance with the results, the parts with high stress are optimized. The result showed that the stress intensity on the back of the air spring mounting base is relatively high. This situation can be improved by changing the radius to $40 \mathrm{~mm}$ fillets. The strength of other parts meets the standard requirement and provided the basis for further optimization calculation. The results have laid a foundation for the fatigue strength test of the suspension chassis of high-speed maglev train.
\end{abstract}

Keywords: High-speed Maglev Vehicle, Levitation Chassis, Finite Element Modeling, Static Strength

\section{Introduction}

Maglev train is a modern high-tech rail transit project driven by magnetic levitation force. It realizes the levitation and guidance between the train and the track without contact through electromagnetic force, and then uses the electromagnetic force generated by linear motor to pull the train to run. Maglev trains mean that these trains use the fundamental principle of magnetism to float on rails instead of the old steel wheels and rail trains [1]. Maglev train runs on the track through levitation, so there are strict requirements on the material and weight of the whole structure. The maglev train levitation chassis reduces the weight of the levitation chassis as much as possible on the premise of meeting the strength requirements. In order to solve the contradiction between strength requirements and weight requirements, it is necessary to calculate and analyze the structural strength of the levitation chassis, so as to determine the structure of the bogie reasonably and put forward improvement suggestions on the local structure [2].

The structure of levitation chassis is different from that of traditional bogie. Due to the limitation of materials, riveting and bolt connection are chosen to combine the two parts. In order to make each levitation control unit have decoupling ability, auxiliary elastic parts and hinges are used to realize this function. It is difficult to realize the function of each connected part in the finite element software. For high-speed maglev train levitation chassis, we analyze the connection mode of levitation chassis and secondary suspension part and establish the finite element model with hypermesh software. According to the actual stress condition, a set of load and constraint project is designed. ANSYS finite element analysis software is used to analyze the static strength of levitation chassis under four different working conditions and we put forward feasible suggestions for structure optimization [3]. 


\section{Finite Element Model}

\subsection{Structure Description}

The structure of high-speed maglev car body is divided into the following three parts: maglev bogie (levitation chassis), the secondary suspension and the carriage. The levitation chassis is composed of a beam frame, a suspension frame and a longitudinal connection structure, in which the beam frame includes two beams, a bolster support beam and an air spring support beam. The suspension frame comprises two supporting arms and upper and lower connecting parts. The longitudinal connecting structure includes a longitudinal beam, a hinge and a swinging handle. A beam frame and two suspension frames constitute a suspension frame unit. The two suspension frames constitute a levitation chassis through a longitudinal connection device [4], and each part is connected by bolts and rivets. Different from the traditional concept of bogie, the rubber suspension (electromagnet suspension) in the levitation chassis is primary suspension, and the air spring part of the swing pillow is secondary suspension. The levitation chassis is the key structure connecting the suspension electromagnet, guiding electromagnet, brake electromagnet and the car body, which can realize the decoupling of the structure.

\subsection{Finite Element Modeling}

The main body of the levitation chassis is made of aluminum alloy. The mechanical connection of aluminum alloy mainly includes rivet connection and bolt connection. The whole structure is connected by a large number of rivets and bolts, which is the difficulty of modeling. After a large number of experiments and studies, the connection mode of rivets and bolts is simulated by coupling and constraint equation method [5]. The pre-tightening force of bolts adopts equivalent force method, which makes the calculation fast and efficient, and can correctly reflect the stress condition of the whole levitation chassis. The finite element method is shown in Figure 1. In order to improve the efficiency of finite element simulation, it is usually necessary to simplify the finite element model reasonably, and reduce the number of features of the model by suppressing or deleting those features that have no or little impact on the problem [6].
Solid185 unit is used for meshing. The discrete model has 2332377 nodes and 7610622 units. The finite element model of magnetic levitation chassis is shown in Figure 2.

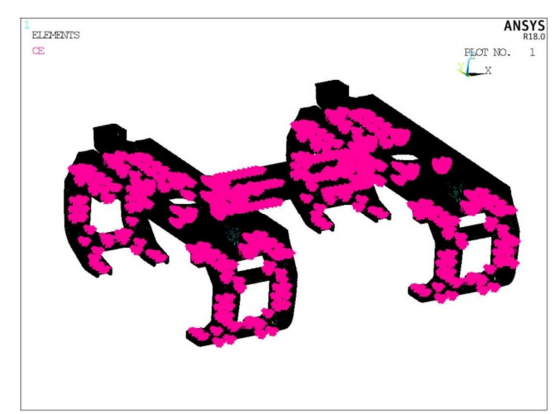

Figure 1. Finite element method for mechanical connection.

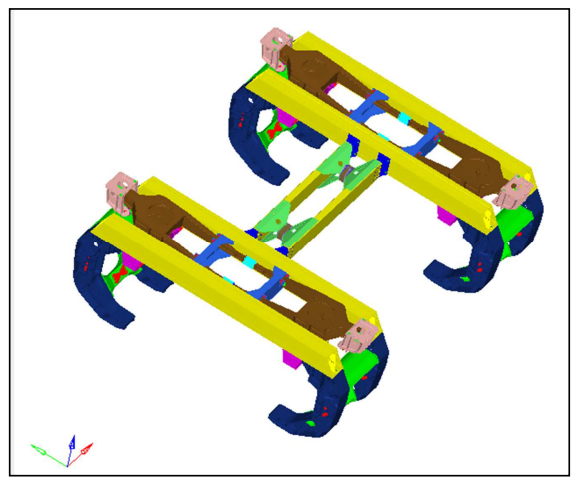

Figure 2. Finite element model of maglev bogie.

\subsection{Material Parameters}

Levitation chassis is mainly made of aluminum alloy with high strength, high elasticity and light density through welding and other processes [7]. Levitation chassis and component materials mainly include EN AC-42100S T6, EN AW-6005A T6, EN AW-6082 T6 and 40CrMo. Among them, as the swing rod is an important connecting structure between the car body and the levitation chassis, as well as the main force transmission component. Thus, $40 \mathrm{CrMo}$ is adopted, and the rest materials are all made of aluminum alloy [8]. The material performance parameters are shown in Table 1.

Table 1. Material performance parameters.

\begin{tabular}{|c|c|c|c|c|}
\hline Material & Elasticity modulus/(MPa) & Poisson's ratio & Density $/\left(\mathrm{kg} \cdot \mathrm{m}^{-3}\right)$ & Yield strength/(MPa) \\
\hline EN AC-42100S T6 & $0.7 \times 10^{5}$ & 0.33 & 2680 & 274 \\
\hline EN AW-6005A T6 & $0.7 \times 10^{5}$ & 0.33 & 2700 & 241 \\
\hline EN AW-6082 T6 & $0.7 \times 10^{5}$ & 0.33 & 2710 & 255 \\
\hline 40 CrMo & $2.06 \times 10^{5}$ & 0.25 & 7900 & 950 \\
\hline
\end{tabular}

\section{Load Condition and Constraint Mode}

Since there is currently no relevant standard for the calculation of maglev levitation chassis, the actual working condition of maglev train dynamics is analyzed to determine the working condition for the calculation of the strength of the levitation chassis. First, the direction principle of the coordinate system is defined. Longitudinal is the direction of lane slip plane. Lateral is the lane slip plane and perpendicular to the driving direction. The vertical direction is perpendicular to the slip plane. This paper will list four typical abnormal working conditions for analysis. 


\subsection{Constraint Mode and Loading}

The function of the levitation chassis is to load electromagnets and transfer the suspension force, guiding force, traction force and braking force to the carriage through the secondary systems [9]. According to the actual operation principle of the levitation chassis, the vertical constraint is at the shaking pillow connected with the car body, the transverse constraint is at the mounting seat of the guiding electromagnet, and the longitudinal constraint is at the connecting hole of the longitudinal beam and the traction rod. According to the stress mode of the levitation chassis during operation, longitudinal and vertical loads are applied to the suspension electromagnet mounting seat, longitudinal and transverse loads are applied to the guiding electromagnet mounting seat, and transverse, longitudinal and vertical loads are applied to the connecting swing rod and apron board [10].

\subsection{Load Working Condition}

According to relevant calculation experience, taking into account such basic conditions as snow load, magnetron dynamic special load, wind load, turning radius, acceleration and so on, four abnormal load conditions under non-fault conditions as shown in Table 2. are formulated. Meanwhile, static equivalence principle is adopted to calculate and analyze the load of each working condition [11-13].

Table 2. Working table for load calculation of maglev bogie.

\begin{tabular}{|c|c|c|c|c|c|}
\hline & & \multicolumn{4}{|c|}{ Abnormal load under non-fault condition } \\
\hline \multicolumn{2}{|l|}{ Working condition } & $\mathrm{S} 3 \mathrm{~A}$ & S5A & S3B & S5B \\
\hline \multicolumn{2}{|l|}{ Snow load } & S-Weight of snow & S- Weight of snow & S- Weight of snow & S- Weight of snow \\
\hline \multicolumn{2}{|l|}{ Acceleration/Braking } & Acceleration & Acceleration & Braking & Braking \\
\hline \multirow{3}{*}{ Acceleration $\left(\mathrm{m} / \mathrm{s}^{2}\right)$} & $a_{x}$ & -1.1 & -1.1 & 1.1 & 1.1 \\
\hline & $\mathrm{a}_{\mathrm{y}}$ & -2.0 & -2.0 & -2.0 & -2.0 \\
\hline & $\mathrm{a}_{\mathrm{z}}$ & 1.2 & 1.2 & 1.2 & 1.2 \\
\hline \multicolumn{2}{|l|}{ Turning Radius } & $\begin{array}{l}\text { Turn left } \\
\mathrm{R}=350 \mathrm{~m}\end{array}$ & Turn left $\mathrm{R}=350 \mathrm{~m}$ & Turn left $\mathrm{R}=1000 \mathrm{~m}$ & Turn left $\mathrm{R}=1000 \mathrm{~m}$ \\
\hline \multicolumn{2}{|l|}{ Wind speed } & $10 \mathrm{~m} / \mathrm{s}$ & $37 \mathrm{~m} / \mathrm{s}$ & $10 \mathrm{~m} / \mathrm{s}$ & $37 \mathrm{~m} / \mathrm{s}$ \\
\hline
\end{tabular}

\section{Analysis of Calculation Results}

Through ANSYS software, according to the above four working conditions to the levitation chassis strength analysis [14]. Generally speaking, for plastic materials such as steel, copper and aluminum, the Von Mises equivalent stress can be selected for strength evaluation according to the fourth strength theory [15]. The overall stress contour of the levitation chassis under four working conditions are shown in Figure 3. respectively.

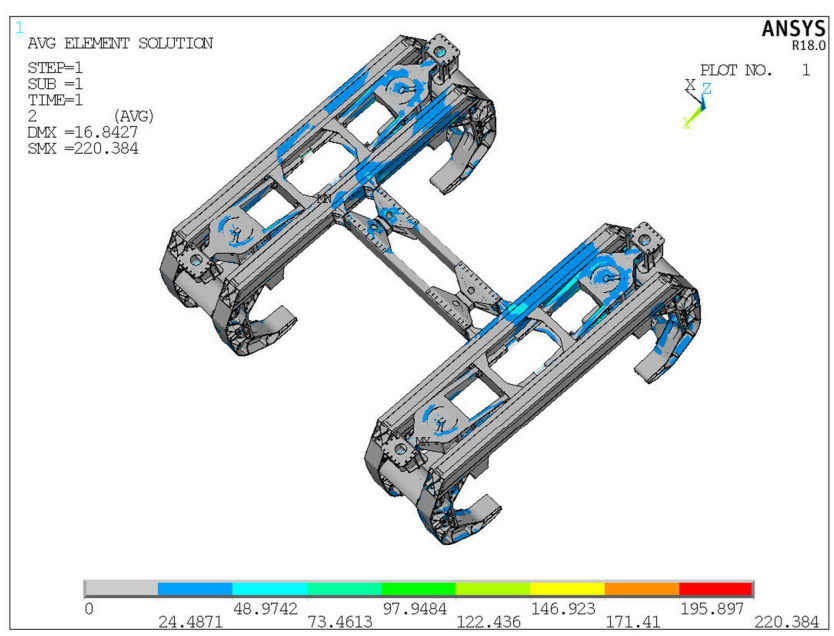

(a) Overall stress distribution diagram of S3A working conditions

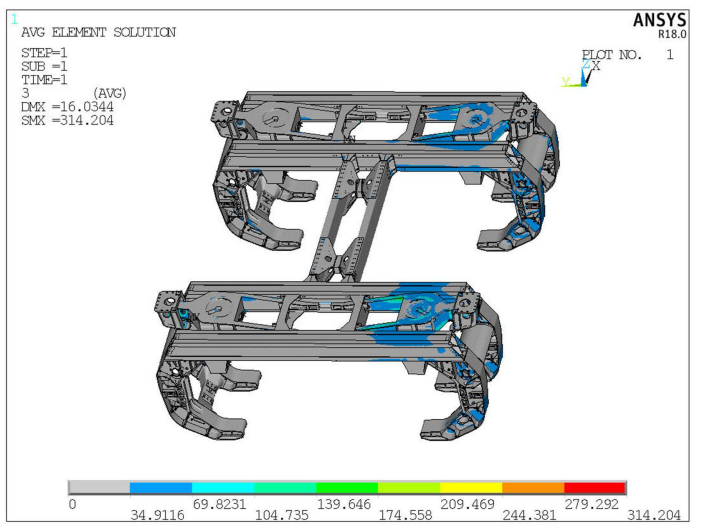

(b) Overall stress distribution diagram of S5A working conditions

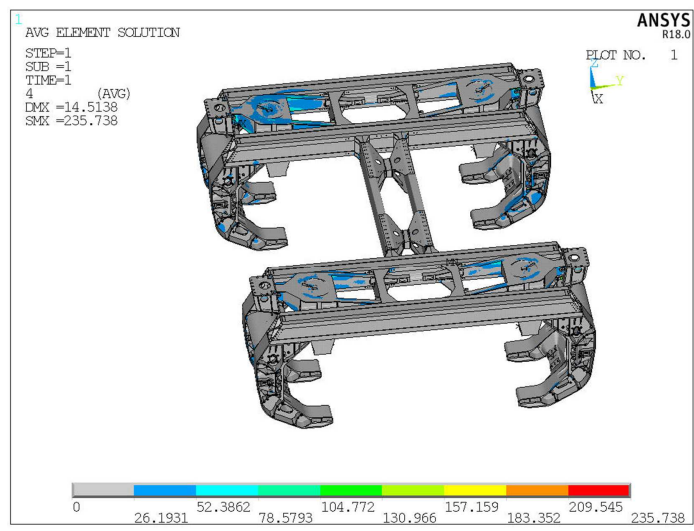

(c) Overall stress distribution diagram of S3B working conditions 


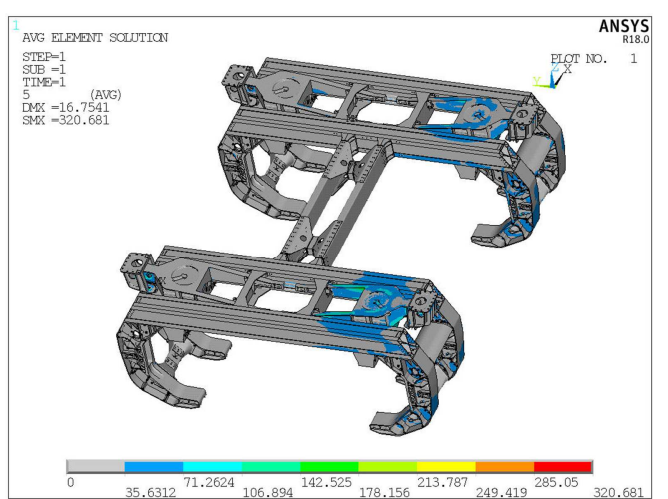

(d) Overall stress distribution diagram of S5B working conditions

Figure 3. Overall stress distribution diagram of maglev bogie.

The maximum stress occurs at the swing rod connected with the secondary suspension and the car body, and the sublarge stress occurs at the stiffened plate at the back of the air spring. Taken S5B in the most severe working condition as an example, the stress distribution at the mounting seat of the air spring is shown in Figure 4.

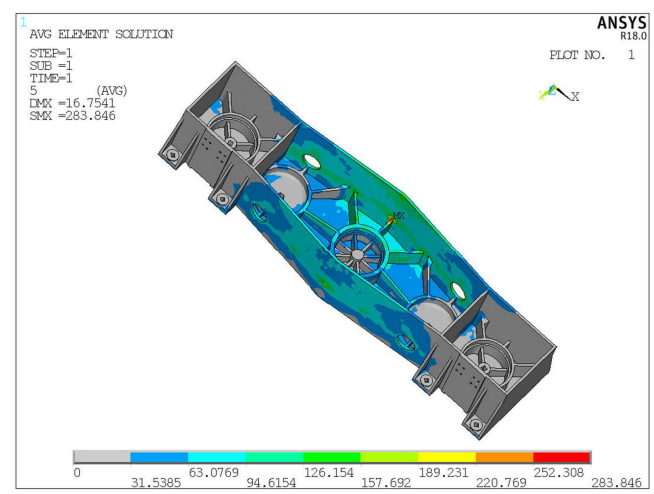

Figure 4. Location of sublarge stress occurring in S5B working conditions.

The connection between the stiffened plate at the back of the air spring mounting seat and the vertical plate is optimized into fillet, and fillet is initially chosen as $\mathrm{R}=10 \mathrm{~mm}$ to replace the previous structure. Taken S5B as an example, finite element strength analysis is carried out after structural optimization. The stress distribution of air spring mounting seat is shown in Figure 5.

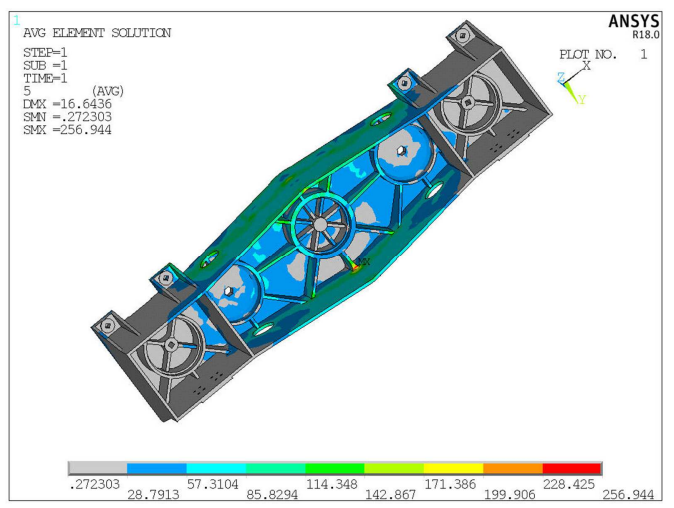

Figure 5. The place where the large stress occurs at the fillet $R=10 \mathrm{~mm}$ under S5B condition.
The corner at the connection between the stiffened plate at the back of the air spring mounting seat and the vertical plate was expanded from $R=10 \mathrm{~mm}$ to $\mathrm{R}=40 \mathrm{~mm}$. Still taken $\mathrm{S} 5 \mathrm{~B}$ as an example, the stress distribution at the air spring mounting seat was shown in Figure 6.

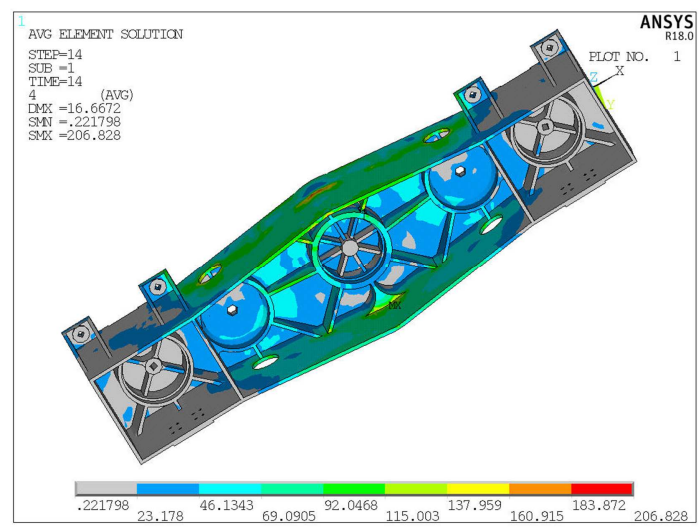

Figure 6. The place where the large stress occurs at the fillet $R=40 \mathrm{~mm}$ under $S 5 B$ condition.

The following conclusions can be drawn from the above figures and tables:

(1) Under the four working conditions, the maximum stress occurs at the swing rod connected with the levitation chassis and the car body, of which the maximum is $320.68 \mathrm{MPa}$ and the material is $40 \mathrm{CrMo}$. The permissible stress of the material is selected and the static strength of the swing rod meets the requirements.

(2) Under the four working conditions, the secondary large stress occurs at the stiffened plate on the back of the air spring mounting seat, and the maximum value is 283.85MPa, which has exceeded the permissible stress of the material and is a serious stress concentration. After the initial optimization of the joint as a fillet of $\mathrm{R}=10 \mathrm{~mm}$, the average stress drops to $256.95 \mathrm{MPa}$. Then we expand the fillet to $\mathrm{R}=40 \mathrm{~mm}$, and the average stress drops to $207.83 \mathrm{MPa}$. After the analysis of the above results, it is suggested that the joint between the stiffened plate and the vertical plate at the back of the air spring mounting seat should be treated as bending corner, and the corner radius of the connection should be increased, so as to reduce the stress value at the back of the air spring mounting seat.

(3) Under the four working conditions, the maximum stress appears under the S5B working condition, indicating that after increasing the turning radius and adding the dynamic special load, the loading condition is more severe and the overall stress of the levitation chassis is worse.

(4) Except that the stress of the stiffened plate at the back of the air spring mounting seat is relatively large, the stress of the rest of the levitation chassis is all less than the yield limit, which meets the requirements of static strength. 


\section{Conclusion}

In this paper, according to the structural characteristics of levitation chassis of the high-speed maglev train with a speed of $600 \mathrm{~km} / \mathrm{h}$ designed by CRRC Qingdao Sifang co., Ltd., hypermesh and ANSYS software are used to design a modeling scheme for the mechanical connection mode and the secondary suspension, and the static strength is calculated according to four abnormal working conditions.

The structure of levitation chassis of the high-speed maglev train with a speed of $600 \mathrm{~km} / \mathrm{h}$ and the finite element analysis results show that suspended load, guide load, drive load and brake load during normal operation is passed on to the bracket through magnet connection device, and then to the car body by the air spring, because the levitation chassis from the magnet and slide force is passed on to the secondary suspension and car body. Thus, under the four working conditions, the stress concentration on the stiffened plate at the back of the air spring mounting seat is serious. It is suggested that the joint should be changed into fillet which can decrease the stress of the stiffened plate at the back of the air spring mounting seat. The rest parts all meet the static strength requirements of their materials, which provides the basis for the subsequent structural optimization.

Meanwhile, this paper provides a constraint mode and loading mode for finite element calculation of levitation chassis of the high-speed maglev train, which can be used to evaluate the stress intensity of levitation chassis, laying a simulation foundation for the fatigue strength test of levitation chassis of the high-speed maglev train.

\section{Acknowledgements}

This work was financially supported by National Key Technologies R\&D Program of China (No. 2016YFB1200602).

\section{References}

[1] Shang Yuejin. Construction and Design of EMU Vehicles [M]. Lanzhou: Lanzhou Jiaotong University, 2010.

[2] Yang Lei, Zhao Zhisu. The Finite Element Analysis on
Structure Strength of the Maglev Bogie [J]. Mechanics, 2004 (02): $13-15+30$.

[3] Li Guoqiang, Wang Zhilu, Chen Suwen, Xu Youlin. Field Measurements and Analyses of Environmental Vibrations Induced by High-speed Maglev [J]. Science of the Total Environment, 2016

[4] Hao Feng. Finite Element Analysis of Key Parts of Running Mechanism of High-speed Maglev Train [D]. National University of Defense Technology, 2002.

[5] Du Pingan. The Basic Principle of Finite Element Meshing [J]. Liaoning: Mechanical Design and Manufacturing, 2000, No. A: 34-36.

[6] Yao Yuan. Study on Air Springs of High-speed Maglev Train [D]. Dalian Jiaotong University, 2008.

[7] Xie Weimin. Dynamical Vehicle-guideway Coupling Emulation Model of Maglev Train System [D]. Southwest Jiaotong University, 2005.

[8] Zhao Weihua. Calculation and Analysis of the Static Strength of the Car Body and its test [J]. Information Recording Materials, 2016, 17 (3): 79-81.

[9] Wang Jianyi. Study on Maglev Interaction of High-speed Maglev Vehicle [D]. Dalian Jiaotong University, 2008.

[10] Santecchia E, Hamouda A M S, Musharavati F, et al. A Review on Fatigue Life Prediction Methods for Metals [J]. Advances in Materials Science and Engineering, 2016, 2016: 1-26.

[11] Ye Xueyan. Modeling and Simulation Analysis of Maglev Vehicle System Dynamics [D]. Southwest Jiaotong University, 2007.

[12] Yu Hualii, Xu Chuangwen. Finite Element Analysis of Drillwell Hook Using Pro/E and ANSYS [J]. Manufacturing Automation, 2009, 31 (10): 119-121.

[13] Ge jun, Gao Dinggang, Zheng Shubin, Cha Xiaodong. Dynamic Load Test and Analysis for High-speed Maglev Vehicle Moving Machine [J]. Journal of Shanghai University of Engineering Science, 2015, 29 (04): 289-292+297.

[14] Hong Qingzhang. Examplar-based Learning of ANSYS [M]. Beijing: China Railway Publishing House, 2002.

[15] Liu Hongwen Mechanics of Materials [M]. Beijing: Higher Education Press, 2007. 